\title{
An Improved Bushell-Okarasinski Type Inequality for Sugeno Integrals
}

\section{Dug Hun Hong}

Department of Mathematics, Myongji University, Yongin, Korea

\section{]jfis}

\begin{abstract}
Recently, Roman-Flores et al. (2008) proposed a Bushell-Okrasinski type inequality for fuzzy integrals. In this paper, we improve the result of Roman-Flores et al. by findings the optimal constant $H_{s}$ for which the following Bushell-Okrasinski type inequality for fuzzy integrals $H_{s}\left((S) \int_{0}^{1}(1-t)^{s-1} g(t)^{s} d \mu\right) \geq\left((S) \int_{0}^{1} g(t) d \mu\right)^{s}$ holds, where $s \geq 1$, $g:[0,1] \rightarrow[0, \infty)$ is a non-increasing function. The case of nondecreasing function is also treated. The result of Roman-Flores et al. is a special case of our result.
\end{abstract}

Keywords: Fuzzy measure, Sugeno integral, Bushell-Okrasinski type inequality

\section{Introduction and Preliminaries}

A number of studies have examined the Sugeno integral since its introduction in 1974 [1]. Ralescu and Adams [2] generalized a range of fuzzy measures and gave several equivalent definitions of fuzzy integrals. Wang and Klir [3] provided an overview of fuzzy measure theory.

Caballero and Sadarangani [4-6] proved a Hermite-Hadamarda type inequality, a Cauchy type inequality, and Fritz Carlson's inequality for fuzzy integrals. Roman-Flores et al. [7- -10 ] presented several new types of inequalities for Sugeno integrals, including a Prekopa-Leindler type inequality, a Jensen type inequality, a Young type inequality, and some convolution type inequalities. Flores-Franulic et al. [11, 12] presented Chebyshev's inequality and Stolarsky's inequality for fuzzy integrals. Ouyang and Fang [13] generalized their main results to prove

Received: Sep. 27, 2018

Revised : Apr. 11, 2020

Accepted: Apr. 24, 2020

Correspondence to: Dug Hun Hong (dhhong@mju.ac.kr)

@The Korean Institute of Intelligent Systems

cc) This is an Open Access article distributed under the terms of the Creative Commons Attribution Non-Commercial License (http://creativecommons.org/licenses/ by-nc/3.0// which permits unrestricted noncommercial use, distribution, and reproduction in any medium, provided the original work is properly cited. some optimal upper bounds for the Sugeno integral of the monotone function in [9]. Ouyang et al. [14] generalized a Chebyshev type inequality for the fuzzy integral of monotone functions based on an arbitrary fuzzy measure. Hong [15] extended previous research by presenting a Hardy-type inequality for Sugeno integrals, Hong [16] proposed a Liapunov type inequality for Sugeno integrals, and Hong [17] proposed a Berward and Favard type inequalities for fuzzy integrals. Hong et al. [19] considered Steffensen's integral inequality for Sugeno integral. Recently, Roman-Flores et al. [10] showed a Bushell-Okrasinski type inequality for fuzzy integrals, but this inequality is not optimal. In this paper, we improve the results of Roman-Flores et al. [10] for $s \geq 1$. Specifically, we find an optimal constant for which a Bushell-Okrasinski type inequality for Sugeno integrals holds for non-increasing functions.

Definition 1. Let $\Sigma$ be a $\sigma$-algebra of subsets of $\mathbb{R}$ and let $\mu: \Sigma \rightarrow[0, \infty]$ be a nonnegative, extended real-valued set function. We say that $\mu$ is a fuzzy measure if and only if 
(a) $\mu(\emptyset)=0$.

(b) $E, F \in \Sigma$ and $E \subseteq F$ imply $\mu(E) \leq \mu(F)$ (monotonicity).

(c) $\left\{E_{p}\right\} \subseteq \Sigma$ and $E_{1} \subseteq E_{2} \subseteq \cdots$ imply $\lim _{p \rightarrow \infty} \mu\left(E_{p}\right)=$ $\mu\left(\bigcup_{p=1}^{\infty} E_{p}\right)$ (continuity form below).

(d) $\left\{E_{p}\right\} \subseteq \Sigma, E_{1} \supseteq E_{2} \supseteq \cdots$, and $\mu\left(E_{1}\right)<\infty$ imply $\lim _{p \rightarrow \infty} \mu\left(E_{p}\right)=\mu\left(\bigcap_{p=1}^{\infty} E_{p}\right)$ (continuity form above).

If $f$ is a non-negative real-valued function defined on $\mathbb{R}$, then we denote by $F_{\alpha}=\{x \in X \mid f(x) \geq \alpha\}=\{f \geq \alpha\}$ the $\alpha$ level of $f$, for $\alpha>0$, and $F_{0}=\overline{\{x \in X \mid f(x)>0\}}=\operatorname{supp}(f)$ is the support of $f$.

We note that

$$
\alpha \leq \beta \Rightarrow\{f \geq \beta\} \subseteq\{f \geq \alpha\}
$$

If $\mu$ is a fuzzy measure on $A \subset \mathbb{R}$, then we define the following:

$$
\mathfrak{F}^{\mu}(A)=\{f: A \rightarrow[0, \infty) \mid f \text { is } \mu \text {-measurable }\}
$$

Definition 2. Let $\mu$ be a fuzzy measure on $(\mathbb{R}, \Sigma)$. If $f \in$ $\mathfrak{F}^{\mu}(\mathbb{R})$ and $A \in \Sigma$, then the Sugeno integral (or the fuzzy integral) of $f$ on $A$, with respect to the fuzzy measure $\mu$, is defined as

$$
\text { (S) } \int_{A} f d \mu=\sup _{\alpha \in[0, \infty)}\left[\alpha \wedge \mu\left(A \cap F_{\alpha}\right)\right] .
$$

In particular, if $A=X$ then

$$
\text { (S) } \int_{\mathbb{R}} f d \mu=(S) \int f d \mu=\sup _{\alpha \in[0, \infty)}\left[\alpha \wedge \mu\left(F_{\alpha}\right)\right] \text {. }
$$

The following properties of the Sugeno integral are well known and can be found in [3]:

Proposition 1 [3]. If $\mu$ is a fuzzy measure on $\mathbb{R}$ and $f, g \in$ $\mathfrak{F}^{\mu}(\mathbb{R})$, then

(i) $(S) \int_{A} f d \mu \leq \mu(A)$;

(ii) $(S) \int_{A} K d \mu=K \wedge \mu(A)$ for any constant $K \in[0, \infty)$;

(iii) $(S) \int_{A} f d \mu \leq(S) \int_{A} g d \mu$, if $f \leq g$ on $A$;

(iv) $\mu(A \cap\{f \geq \alpha\}) \geq \alpha \Rightarrow(S) \int_{A} f d \mu \geq \alpha$;

(v) $\mu(A \cap\{f \geq \alpha\}) \leq \alpha \Rightarrow(S) \int_{A} f d \mu \leq \alpha$; (vi) $(S) \int_{A} f d \mu<\alpha \Leftrightarrow$ there exists $\gamma<\alpha$ such that $\mu(A \cap\{f \geq \gamma\})<\alpha$

(vii) $(S) \int_{A} f d \mu>\alpha \Leftrightarrow$ there exists $\gamma>\alpha$ such that $\mu(A \cap\{f \geq \gamma\})>\alpha$.

Note 1. Let $F(\alpha)=\mu(A \cap\{f \geq \alpha\})$. Then by Proposition 1, (iv), (v),

$$
F(\alpha)=\alpha \Rightarrow(S) \int_{0}^{1} f(x) d \mu=\alpha .
$$

Theorem 1 [13]. Let $f:[0, \infty) \rightarrow[0, \infty)$ be continuous and non-increasing or non-decreasing functions and $\mu$ be the Lebesgue measure on $\mathbb{R}$. Let $(S) \int_{0}^{a} f(x) d \mu=p$. If $0<p<$ $a$, then $f(p)=p$ and $f(a-p)=p$, respectively.

\section{Bushell-Okrasinski Type Inequality}

The classical Bushell-Okrasinski inequality provides the following inequality [19]:

$$
\int_{0}^{x}(x-t)^{s-1} g(t)^{s} d \mu \leq\left(\int_{0}^{x} g(t) d \mu\right)^{s}, \quad 0 \leq x \leq b,
$$

where $1 \leq s, g:[0,1] \rightarrow[0, \infty)$ is a continuous and increasing function. After changing the variable $t=s x$, Malamud [20] analyzed the Bushell-Okrasinski inequality in the following new form:

$$
s \int_{0}^{1}(1-t)^{s-1} g(t)^{s} d \mu \leq\left(\int_{0}^{1} g(t) d \mu\right)^{s} .
$$

Recently, Roman-Flores et al. [10] showed a Bushell-Okrasinski inequality derived from (1) for Sugeno integrals as follows:

Theorem 2 (Roman-Flores et al.) [10]. Let $g:[0,1] \rightarrow[0, \infty)$ be a continuous decreasing function and let $\mu$ be the Lebesgue measure on $\mathbb{R}$. Then

$$
s(S) \int_{0}^{1}(1-t)^{s-1} g(t)^{s} d \mu \geq\left((S) \int_{0}^{1} g(t) d \mu\right)^{s}
$$

holds for all $2 \leq s$

We now improve the above result.

Theorem 3 (Fuzzy Bushell-Okrasinski inequality). Let $g$ : $[0,1] \rightarrow[0, \infty)$ be a continuous non-increasing function and let $\mu$ be the Lebesgue measure on $\mathbb{R}$. Then

$$
\left((S) \int_{0}^{1}(1-t)^{s-1} d \mu\right)^{-1}\left((S) \int_{0}^{1}(1-t)^{s-1} g(t)^{s} d \mu\right)
$$




$$
\geq\left((S) \int_{0}^{1} g(t) d \mu\right)^{s}
$$

holds for all $1 \leq s$.

Proof. Let

$$
H_{s}=\sup \left\{\frac{\left((S) \int_{0}^{1} g(t) d \mu\right)^{s}}{(S) \int_{0}^{1}(1-t)^{s-1} g(t)^{s} d \mu} \mid g \in \mathcal{G}\right\},
$$

where $\mathcal{G}$ is the set of functions which are non-increasing on $[0,1]$ and let

$$
H_{s}(\alpha)=\sup \left\{\frac{\alpha^{s}}{(S) \int_{0}^{1}(1-t)^{s-1} g(t)^{s} d \mu} \mid g \in \mathcal{G}_{\alpha}\right\},
$$

where $\mathcal{G}_{\alpha}=\left\{g \in \mathcal{G} \mid(S) \int_{0}^{1} g(t) d \mu=\alpha\right\}$ for $\alpha \in[0,1]$. Then

$$
H_{s}=\sup _{0<\alpha \leq 1} H_{s}(\alpha) .
$$

We consider $H_{s}(\alpha)$. Let

$$
g_{0}(x)= \begin{cases}\alpha, & \text { if } x \in[0, \alpha)) \\ 0, & \text { if } x \in[\alpha, 1]\end{cases}
$$

Because $\mu\left\{g_{0} \geq \alpha\right\}=\alpha$, by Note 1

$$
(S) \int_{0}^{1} g_{0}(x) d \mu=\alpha .
$$

Then it is easy to check that $g_{0}=\inf \mathcal{G}_{\alpha}$. Thus, we have

$$
H_{s}(\alpha)=\frac{\alpha^{s}}{(S) \int_{0}^{1}(1-t)^{s-1} g_{0}(t)^{s} d \mu} .
$$

Now, let

$$
\text { (S) } \int_{0}^{1}(1-t)^{s-1} g_{0}(t)^{s} d \mu=t_{0}
$$

Because $g_{0}$ is continuous and decreasing on $[0, \alpha]$ and the left limit of $g_{0}$ at $\alpha$ is less than $\alpha$, by Theorem 1 ,

$$
t_{0}=\left(1-t_{0}\right)^{s-1} \alpha^{s}
$$

To find $H_{s}$ we now consider the following optimization problem:

$$
H_{s}=\sup _{0<\alpha \leq 1} H_{s}(\alpha)=\text { Maximize } \frac{\alpha^{s}}{t}
$$

where

$$
t=(1-t)^{s-1} \alpha^{s}, \quad 0<\alpha \leq 1 .
$$

We first show that $H_{s}(\alpha)=\frac{\alpha^{s}}{t}$ is an increasing function of $\alpha$. We have

$$
H_{s}^{\prime}(\alpha)=\frac{\alpha^{s-1}}{t^{2}}\left(s t-\alpha \frac{d t}{d \alpha}\right)
$$

From the equation $t=(1-t)^{s-1} \alpha^{s}$, we now have

$$
\begin{aligned}
s \alpha^{s-1} & =\frac{(1-t)^{s-1}+t(s-1)(1-t)^{s-2}}{(1-t)^{2 s-2}} \frac{d t}{d \alpha} \\
& =\frac{1+t(s-1)(1-t)^{-1}}{(1-t)^{s-1}} \frac{d t}{d \alpha},
\end{aligned}
$$

which implies that

$$
\alpha \frac{d t}{d \alpha}=\frac{1}{1+t(s-1)(1-t)^{-1}} s t \leq s t .
$$

Then $H_{s}^{\prime}(\alpha) \geq 0$, that is, $H_{s}$ is nondecreasing and thus

$$
H_{s}=H_{s}(1)=\frac{1}{(S) \int_{0}^{1}(1-t)^{s-1} d \mu},
$$

which completes the proof.

Note 2. As shown in the proof of Theorem 3, the continuity assumption of $g$ is not needed.

Note 3. If $g(t)=1$ in the inequality of Theorem 3, then the equality holds. Therefore, we see that the inequality in Theorem 3 is optimal.

Lemma 1. Let $(S) \int_{0}^{1}(1-t)^{s-1} d \mu=x^{*}, s \geq 2$. Then

$$
\frac{1}{s} \leq \frac{1}{s}\left(\frac{2(s-1)^{s-1}}{(s-1)^{s-1}+s^{s-2}}\right)<x^{*} .
$$

Proof. Suppose that $f(t)=(1-t)^{s-1}-t$. Then by Theorem $1, f\left(x^{*}\right)=0$. We note that

$$
f^{\prime}(t)=-(s-1)(1-t)^{s-2}-1<0, \quad f^{\prime}(0)=-s,
$$

and

$$
f^{\prime \prime}(t)=(s-1)(s-2)(1-t)^{s-3} \geq 0 .
$$

Because $f$ is decreasing and convex, we have

$$
\frac{1}{s}=-\frac{f(0)}{f^{\prime}(0)}<x^{*}
$$


and similarly, we have

$$
\frac{1}{s} \leq \frac{1}{s}-\frac{f\left(\frac{1}{s}\right)}{f^{\prime}\left(\frac{1}{s}\right)}<x^{*}
$$

and

$$
\begin{array}{rlrl}
\frac{1}{s}-\frac{f\left(\frac{1}{s}\right)}{f^{\prime}\left(\frac{1}{s}\right)} & = & \frac{1}{s}-\frac{\left(1-\frac{1}{s}\right)^{s-1}-\frac{1}{s}}{1-(s-1)\left(1-\frac{1}{s}\right)^{s-2}} \\
& =\quad \frac{1}{s}\left(\frac{2(s-1)^{s-1}}{(s-1)^{s-1}+s^{s-2}}\right),
\end{array}
$$

which completes the proof.

The following result of Roman-Flores et al. [10] is a special case of our results.

Corollary 1 (Roman-Flores et al. [?]). Let $g:[0,1] \rightarrow$ $[0, \infty)$ be a non-increasing function and let $\mu$ be the Lebesgue measure on $\mathbb{R}$. Then

$$
s\left((S) \int_{0}^{1}(1-t)^{s-1} g(t)^{s} d \mu\right) \geq\left((S) \int_{0}^{1} g(t) d \mu\right)^{s}
$$

holds for all $2 \leq s$.

Corollary 2. Let $g:[0,1] \rightarrow[0, \infty)$ be a non-increasing function and that $\mu$ is the Lebesgue measure on $\mathbb{R}$. Then

$$
\begin{aligned}
& s\left(\frac{(s-1)^{s-1}+s^{s-2}}{2(s-1)^{s-1}}\right)\left((S) \int_{0}^{1}(1-t)^{s-1} g(t)^{s} d \mu\right) \\
& \geq\left((S) \int_{0}^{1} g(t) d \mu\right)
\end{aligned}
$$

holds for all $2 \leq s$.

Example 1. We compare our result with that of RomanFlores et al. [10] for $s=2,3,4,5,10,20$. That is,

\begin{tabular}{cc}
\hline$s$ & $\left((S) \int_{0}^{1}(1-t)^{s-1} d \mu\right)^{-1}$ \\
\hline 2 & 2.000 \\
3 & 2.618 \\
4 & 3.148 \\
5 & 3.630 \\
10 & 5.692 \\
20 & 9.110 \\
\hline
\end{tabular}

Our results indicates much lower than that of Roman-Flores et al. [10].

The case of a non-increasing function is similar.

Theorem 4. Let $g:[0,1] \rightarrow[0, \infty)$ be a non-decreasing function and let $\mu$ be the Lebesgue measure on $\mathbb{R}$. Then

$$
\begin{aligned}
& \left((S) \int_{0}^{1} t^{s-1} d \mu\right)^{-1}\left((S) \int_{0}^{1} t^{s-1} g(t)^{s} d \mu\right) \\
& \geq\left((S) \int_{0}^{1} g(t) d \mu\right)^{s}
\end{aligned}
$$

holds for all $1 \geq s$

\section{Conflict of Interest}

No potential conflict of interest relevant to this article was reported.

\section{Acknowledgements}

This research was supported by Basic Science Research Program through the National Research Foundation of Korea (NRF) funded by the Ministry of Education (2017R1D1A1B027869).

\section{References}

[1] M. Sugeno, "Theory of fuzzy integrals and its applications,'Ph.D. dissertation, Tokyo Institute of Technology, Tokyo, Japan, 1974.

[2] D. Ralescu and G. Adams, "The fuzzy integral," Journal of Mathematical Analysis and Applications, vol. 75, no. 2, pp. 562-570, 1980. https://doi.org/10.1016/0022-247X(80) 90101-8

[3] Z. Wang and G. J. Klir, Fuzzy Measure Theory. New York, NY: Plenum, 1992.

[4] J. Caballero and K. Sadarangani, "Hermite-Hadamard inequality for fuzzy integrals," Applied Mathematics and Computation, vol. 215, no. 6, pp. 2134-2138, 2009. https: //doi.org/10.1016/j.amc.2009.08.006

[5] J. Caballero and K. Sadarangani, "Fritz Carlson's inequality for fuzzy integrals," Computers \& Mathematics with Applications, vol. 59, no. 8, pp. 2763-2767, 2010. https: //doi.org/10.1016/j.camwa.2010.01.045

[6] J. Caballero and K. Sadarangani, "A Cauchy-Schwarz type inequality for fuzzy integrals," Nonlinear Analysis: Theory, Methods and Applications, vol. 73, no. 10, pp. 3329-3335, 2010. https://doi.org/10.1016/j.na.2010.07.013 
[7] H. Roman-Flores, A. Flores-Franulic, and Y. Chalco-Cano, “A Hardy-type inequality for fuzzy integrals," Applied Mathematics and Computation, vol. 204, no. 1, pp. 178-183, 2008. https://doi.org/10.1016/j.amc.2008.06.027

[8] H. Roman-Flores, A. Flores-Franulic, and Y. Chalco-Cano, "A Jensen type inequality for fuzzy integrals," Information Sciences, vol. 177, no. 15, pp. 3192-3201, 2007. https: //doi.org/10.1016/j.ins.2007.02.006

[9] H. Roman-Flores, A. Flores-Franulic, and Y. Chalco-Cano, "The fuzzy integral for monotone functions," Applied Mathematics and Computation, vol. 185, no. 1, pp. 492-498, 2007. https://doi.org/10.1016/j.amc.2006.07.066

[10] H. Roman-Flores, A. Flores-Franulic, and Y. ChalcoCano, "A convolution type inequality for fuzzy integrals," Applied Mathematics and Computation, vol. 195, no. 1, pp. 94-99, 2008. https://doi.org/10.1016/j.amc.2007.04.072

[11] A. Flores-Franulic and H. Roman-Flores, "A Chebyshev type inequality for fuzzy integrals," Applied Mathematics and Computation, vol. 190, no. 2, pp. 1178-1184, 2007. https://doi.org/10.1016/j.amc.2007.02.143

[12] A. Flores-Franulic, H. Roman-Flores, and Y. ChalcoCano, "A note on fuzzy integral inequality of Stolarsky type," Applied Mathematics and Computation, vol. 196, no. 1 pp. 55-59, 2008. https://doi.org/10.1016/j.amc.2007.05. 032

[13] Y. Ouyang and J. Fang, "Sugeno integral of monotone functions based on Lebesgue measure," Computers \& Mathematics with Applications, vol. 56, no. 2, pp. 367-374, 2008. https://doi.org/10.1016/j.camwa.2007.11.044

[14] Y. Ouyang, J. Fang, and L. Wang, "Fuzzy Chebyshev type inequality," International Journal of Approximate Reasoning, vol. 48, no. 3, pp. 829-835, 2008. https://doi.org/10. 1016/j.ijar.2008.01.004

[15] D. H. Hong, "A sharp Hardy-type inequality of Sugeno integrals," Applied Mathematics and Computation, vol. 217, no. 1, pp. 437-440, 2010. https://doi.org/10.1016/j.amc 2010.05 .071

[16] D. H. Hong, "A Liapunov type inequality for Sugeno integrals," Nonlinear Analysis: Theory, Methods and Applications, vol. 74, no. 18, pp. 7296-7303, 2011. https: //doi.org/10.1016/j.na.2011.07.046

[17] D. H. Hong, "Berwald and Favard type inequalities for fuzzy integrals," International Journal of Uncertainty, Fuzziness and Knowledge-Based Systems, vol. 24, no. 1, pp. 47-58, 2016. https://doi.org/10.1142/S0218488516500033

[18] D. H. Hong, E. L. Moon, and J. D. Kim, "Steffensen's integral inequality for the Sugeno integral," International Journal of Uncertainty, Fuzziness and Knowledge-Based Systems, vol. 22, no. 2, pp. 235-241, 2014. https://doi.org/ $10.1142 / \mathrm{S} 0218488514500111$

[19] P. J. Bushell and W. Okrasinski, "Nonlinear Volterra integral equations with convolution kernel," Journal of the London Mathematical Society, vol. s2-41, no. 3, pp. 503510, 1990. https://doi.org/10.1112/jlms/s2-41.3.503

[20] S. M. Malamud, "Some complements to the Jensen and Chebyshev inequalities and a problem of W. Walter," Proceedings of the American Mathematical Society, vol. 129, pp. 2671-2678, 2001. https://doi.org/10.1090/S0002-993901-05849-X

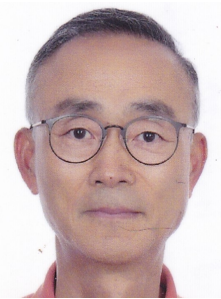

Dug Hun Hong received the B.S. and M.S. degrees in Mathematics from Kyungpook National University, Taegu Korea in 1981 and 1983, respectively. He received the M.S. and Ph.D. degrees from the University of Minnesota in 1988 and 1990, respectively. His research interests include probability theory and fuzzy theory with applications.

E-mail: dhhong@mju.ac.kr 